\title{
Hybrid Assistive Limb Functional Treatment for a Patient with Chronic Incomplete Cervical Spinal Cord Injury
}

\author{
Yuichiro Soma' \\ Shigeki Kubota ${ }^{2}$ \\ Hideki Kadone ${ }^{3}$ \\ Yukiyo Shimizu' \\ Hiroshi Takahashi (iD) ${ }^{2}$ \\ Yasushi Hada' \\ Masao Koda ${ }^{2}$ \\ Yoshiyuki Sankai ${ }^{4}$ \\ Masashi Yamazaki ${ }^{2}$
}

'Department of Rehabilitation Medicine, University of Tsukuba Hospital, Ibaraki, Japan; ${ }^{2}$ Department of Orthopaedic

Surgery, Faculty of Medicine, University of Tsukuba, Ibaraki, Japan; ${ }^{3}$ Center for Innovating Medicine and Engineering (CIME), University of Tsukuba Hospital, Ibaraki, Japan; ${ }^{4}$ Faculty of Systems and Information Engineering, University of Tsukuba, Ibaraki, Japan
Correspondence: Shigeki Kubota Department of Orthopaedic Surgery, Faculty of Medicine, University of

Tsukuba, I-I-I, Tennodai, Tsukuba, Ibaraki, 305-8575, Japan

Tel $+8 \mathrm{I}-29-853-3219$

$\mathrm{Fax}+81-29-853-3162$

Email skubota@tsukuba-seikei.com
Introduction: The hybrid assistive limb (HAL) is a wearable exoskeleton cyborg that assists walking and lower limb movements via real-time actuator control by detecting the wearer's bioelectric signals on the surface of their skin.

Purpose: The purpose of this study was to report the improvement in walking ability following HAL gait training in a patient with tetraplegia after incomplete cervical spinal cord injury (SCI).

Patient and Methods: A 47-year-old man with traumatic cervical SCI for six months after fall had incomplete tetraplegic SCI grade C as classified according to the American Spinal Cord Injury Association impairment scale and was unable to walk in conventional rehabilitation.

Results: The HAL gait training was received 2 or 3 times per week for 13 sessions. Improvement was observed in gait speed (baseline: 0.12; after training: $0.45 \mathrm{~m} / \mathrm{sec}$ ), step length (baseline: 0.30; after training: $0.45 \mathrm{~m}$ ), and cadence (baseline: 23.1 ; after training: $59.6 \mathrm{steps} / \mathrm{min}$ ) based on a 10-meter walking test; International Standards for Neurological and functional Classification of Spinal Cord Injury (ISNCSCI) motor score (baseline: 59; after training: 76); and walking index for spinal cord injury (WISCI) II score (baseline: 1; after training: 6).

Conclusion: We report the recovery of walking ability in a patient with chronic severe incomplete tetraplegic SCI following the HAL training.

Keywords: hybrid assistive limb, robotic rehabilitation, incomplete spinal cord injury, robotic gait training

\section{Introduction}

Spinal cord injury (SCI) is a devastating condition usually associated with permanent impairment. ${ }^{1}$ The majority of neurological recovery from SCI occurs within the first 3 months after the injury. The guidelines to conduct clinical trials for SCI, as developed by The International Campaign for Cures of Spinal Cord Injury Paralysis panel, are spontaneous recovery after SCI and statistical power needed for therapeutic clinical trials. ${ }^{2}$ Beyond this, only a small amount of neurological recovery can be observed, as the neurological functioning is subsequently maintained at a constant level. ${ }^{3}$ Promotion of significant neurological recovery in chronic phase SCI is thought to be difficult, based on animal studies and experience in clinical situations.

Recently, various wearable and mobile exoskeleton devices have been introduced in clinical setting. ${ }^{4}$ The hybrid assistive limb (HAL) robot is a wearable 
exoskeleton cyborg that provides real-time assistance to an individual for walking and limb movements through actuators mounted on the bilateral hip and knee joints. ${ }^{5}$ Lower limb motion is assisted by the detection of bioelectric signals through an electrode on the anterior and posterior surfaces of the wearer's thigh, which are processed through a computer on the back of the HAL. Use of the neurologically controlled exoskeleton HAL leads to functional improvements in the walking abilities of patients in the chronic phase of SCI with residual muscular functions. ${ }^{6}$ Previous pilot studies have reported that body weight support treadmill training with HAL is feasible and safe and can lead to improved functional mobility and motor functions in patients with chronic paraplegia or tetraplegia due to $\mathrm{SCI} .^{7,8}$ To date, the effects of HAL treatment for patients with chronic SCI remain to be elucidated. In this case report, we described a patient with chronic incomplete tetraplegic SCI who showed apparent improvement in walking ability after HAL gait training despite experiencing a more severe impairment in walking ability than those reported in previous studies.

\section{Patients and Methods}

This study was approved by the Ethics Committee of the Tsukuba University Faculty of Medicine (approval no.: H26-22). The patient agreed to participate in this study and provided written informed consent.

\section{Case Presentation}

\section{Course of Medical History in Other Hospitals}

This case report describes a 47-year-old man diagnosed with cervical spondylotic myelopathy who underwent posterior C3-C6 laminoplasty and C7-T1 laminectomy in another hospital. After the surgery, the usual rehabilitation of five sessions per week was started, and improvement in walking ability independently with cane use was observed. After discharge from the other hospital, the patient received rehabilitation 2-3 sessions per week as an outpatient. Two months postoperatively, he fell at his house and was diagnosed with traumatic cervical SCI in the other hospital. The T2-weighted (T2WI) magnetic resonance image (MRI) of his cervical spine showed residual spinal cord compression at the $\mathrm{C} 5 / 6$ level even after decompression surgery. After he fell and was diagnosed with traumatic cervical SCI, the patient had never had surgery and had been treated using conventional rehabilitation in the other hospital. However, due to severe inability to walk, nonsignificant improvement in ambulatory function was observed (Figure 1).

\section{In Our Hospital as an Inpatient}

Six months after the injury, the patient was referred to our hospital to receive rehabilitation training with HAL as an inpatient. His physical condition, activities of daily living, and gait functions before HAL gait training were evaluated. On admission, the abovementioned evaluation revealed that the patient had incomplete tetraplegic SCI grade $\mathrm{C}$ as classified according to the American Spinal Cord Injury Association impairment scale. ${ }^{9}$ According to the International Standards for Neurological and Functional Classification of Spinal Cord Injury (ISNCSCI), ${ }^{9,10}$ the patient's condition was defined with a motor score of 59 points, motor neurological level C7, sensory score for a pinprick was 78 points (right: 39 points, left: 39 points), sensory neurological levels were $\mathrm{T} 10$ on the right and left sides. A Walking Index for Spinal Cord Injury (WISCI) II score of 1 point (the patient needs two assistances to walk a distance of less than 10 meters). ${ }^{11,12}$ The patient's Berg balance scale (BBS) score was 5 points, and his Functional Independence Measure (FIM) motor score was 35 points. Muscle spasticity using a modified Ashworth scale score (mAs) showed that knee extension was 1 point (right: 1 point, left: 0 point) and ankle dorsiflexion were 4 points (right: 2 points, left: 2 points). Deep sensibility of the lower legs was normal; however, the patient had moderate numbness of the lower legs. The clinical progression of the patient is summarized in Figure 1.

\section{HAL Gait Training}

Two therapists and an assistant attached and detached the HAL exoskeleton suit, and an engineer measured gait movement analysis. To maximize safety, a walking device (All-in-One Walking Trainer, Ropox A/S, Naestved, Denmark) with a harness was used to prevent falls during HAL training. All HAL training sessions were performed (including standing, sitting, and walking) with support of the All-in-One Walking Trainer. The HAL training was started 11 months after the patient's surgery and included a total of 13 sessions across 2 months, with 2-3 sessions per week. The duration of one training session was approximately $60 \mathrm{~min}$, including fitting and evaluation. The net gait training time was $15-20 \mathrm{~min}$. This case primarily used the cybernic voluntary control (CVC) mode of the HAL exoskeleton, which allows the operator 


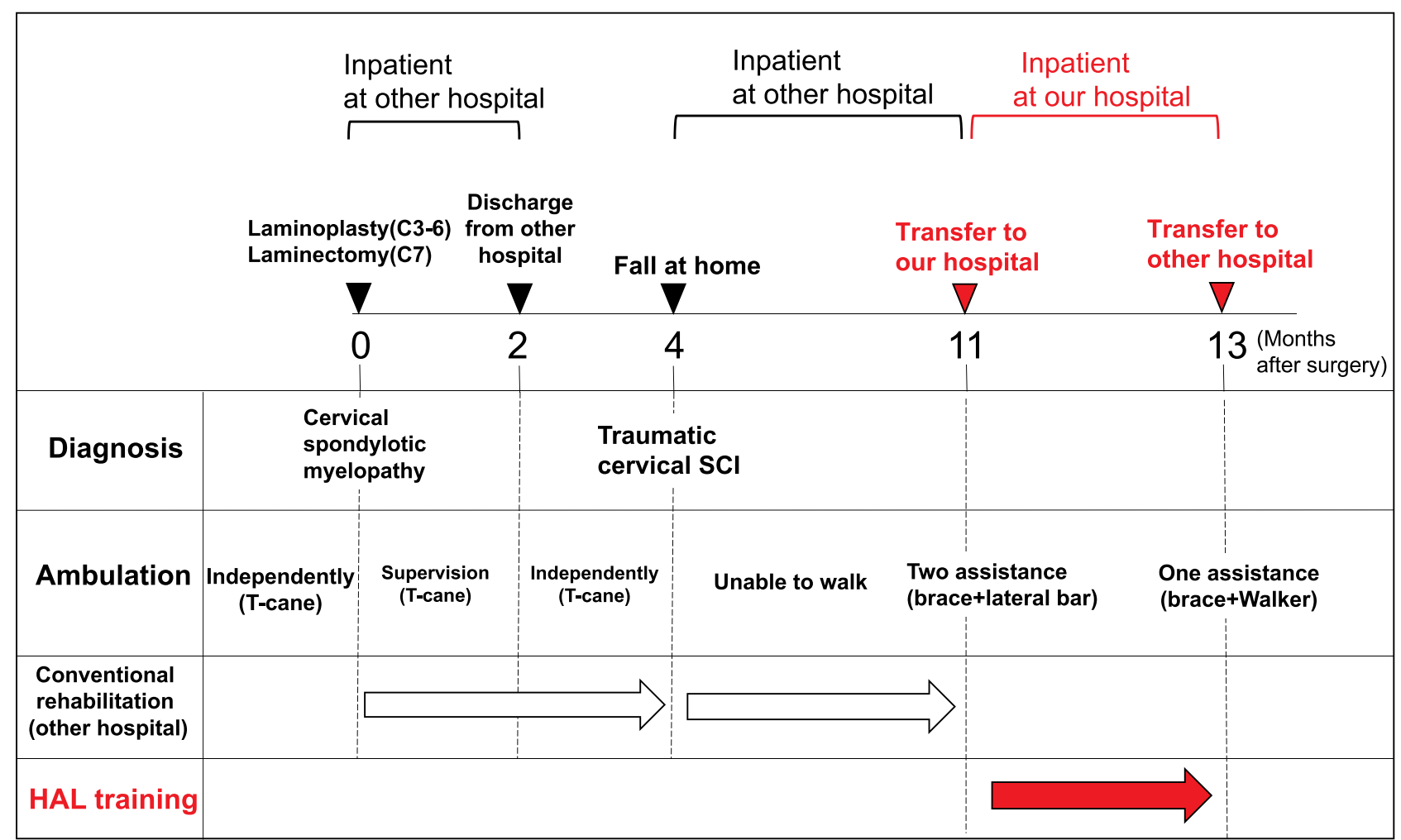

Figure I Postoperative progress during hybrid assistive limb training and conventional rehabilitation at the other hospital, ambulation, and diagnosis.

to adjust the degree of physical support provided for the patient's comfort. The amount of walking distances during the HAL training sessions was decided considering the patient's vital signs and assessing the fatigue and motivation of the patient. Combined conventional rehabilitation was also performed by a physiotherapist and an occupational therapist four times a week (40 min per session).

\section{Assessment}

We evaluated the occurrence of adverse events related to the HAL training. Using the 10-meter walking test (10MWT), we measured walking speed, step length, and cadence in every session. The $10-\mathrm{m}$ walking time was measured using a handheld stopwatch, from which the walking speed was calculated. The number of steps taken between the start and finish line were counted to calculate step length (in meters). Cadence was calculated based on the number of steps taken over the walking time and measured as number of steps/minute. During the 10MWT, the patient was instructed to walk without wearing the robot on a flat surface at a self-selected comfortable pace. The adverse events and 10MWT were evaluated for every session. The ISCNCSI motor score, WISCI-II, BBS, modified Ashworth scale score
(mAs), and FIM were also assessed at baseline (preHAL) and after all HAL sessions were completed (postHAL).

Muscle activity, including that of the rectus femoris, gluteus maximus, and gastrocnemius, during the HAL gait sessions were evaluated using electromyography (EMG) with the Trigno ${ }^{\mathrm{TM}}$ Lab Wireless EMG System (Delsys, Inc., Boston, MA, USA). Each muscle activity was recorded bilaterally using the EMG, collected at 2000 $\mathrm{Hz}$, and filtered with a $30-400 \mathrm{~Hz}$ bandwidth passing filter using scripts on MATLAB 8.2 (Mathworks, Natick, MA, USA). Furthermore, a motion capture system (Vicon MX with 16 T20S cameras, Vicon, Oxford, UK) was used to evaluate the gait motion in synchronization with the muscles' activities. Auto-reflective markers were placed on the legs following the instructions of the VICON plug-in gait marker set at the anterior superior iliac spine, posterior superior iliac spine, lower lateral $1 / 3$ surface of the thigh, flexion-extension axis of the knee, lower lateral $1 / 3$ surface of the shank, head of the second metatarsal bone of the toe, lateral malleolus of the ankle, and posterior peak of the calcaneus of the heel.

Gait motion data were collected at $100 \mathrm{~Hz}$, and the sagittal plane joint angles were computed using the plug-in 
gait model. The kinematic data were extracted from the 10MWT baseline (pre-HAL) and after all HAL sessions were completed (post-HAL). For each of the extracted cycles, the maximum and minimum angles at the hip, knee, and ankle joints were extracted to calculate the joint range of motion. The kinematic profile of each of the lower extremities for each extracted step cycle was normalized to the duration of the step cycle and averaged across cycles.

\section{Results}

Regarding the 10MWT, significant improvements in gait speed (baseline: 0.12; after training: $0.45 \mathrm{~m} / \mathrm{sec}$ ), step length (baseline: 0.30; after training: $0.45 \mathrm{~m}$ ), and cadence (baseline: 23.1; after training: 59.6 steps/min) observed after the HAL training. The HAL walking distance also gradually increased (baseline: 104; after training: $312 \mathrm{~m}$ ) (Figure 2A and $\mathrm{B}$, and video). Other outcomes improved overall, including the ISNCSCI motor score (from 59 to 76 points; with upper extremities from 30 to 38, lower extremities from 29 to 38 ) (Table 1), sensory score for a pinprick (from 78 points to 84 points; right: 41 points, left: 43 points), WISCI-II score (from 1 to 6 , with a progression from using a walker and brace; left side, short leg brace), BBS (from 5 to 7 points, sitting with back unsupported but feet supported on the floor or on a stool and standing to sitting), FIM motor score (from 35 to 39 points, transfers, walking, and grooming), the knee extension (from 1 point to 0 point), and the ankle dorsiflexion of mAs (from 4 points [right: 2 points, left: 2 points] to 3 points [right: 2 points, left: 1 points]).

Muscle activities in the 10MWT baseline (pre-HAL) and after the HAL training (post-HAL) are shown in Figure 3. Each muscle on the right side was activated in the stance phase after the HAL training session. However, the muscle activation on the left side was not changed. Moreover, lower limb kinematics changed after the HAL training (Figure 4). The maximum hip extension angle and knee extension angle on both sides during the stance phases increased after the HAL training sessions. Although the maximum ankle plantar flexion angle on the right side during the stance phases also increased, that on the left side showed no difference between preand post-HAL training. During all HAL sessions, no serious adverse events were related to HAL training directly, except for slight left knee pain after the training. There were moderate swelling and pain in front of the knee (superior border of patella) only after 3 sessions; after

\section{A Walking speed $(\mathrm{m} / \mathrm{sec})$}



B Cadence (steps/min)

Step length $(\mathrm{m})$

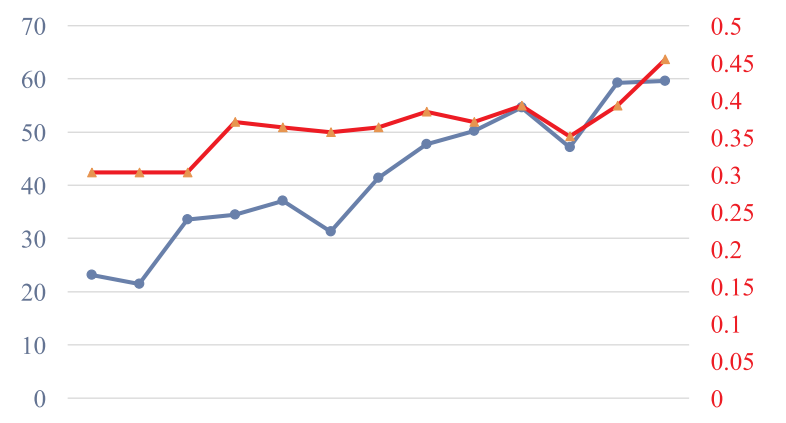

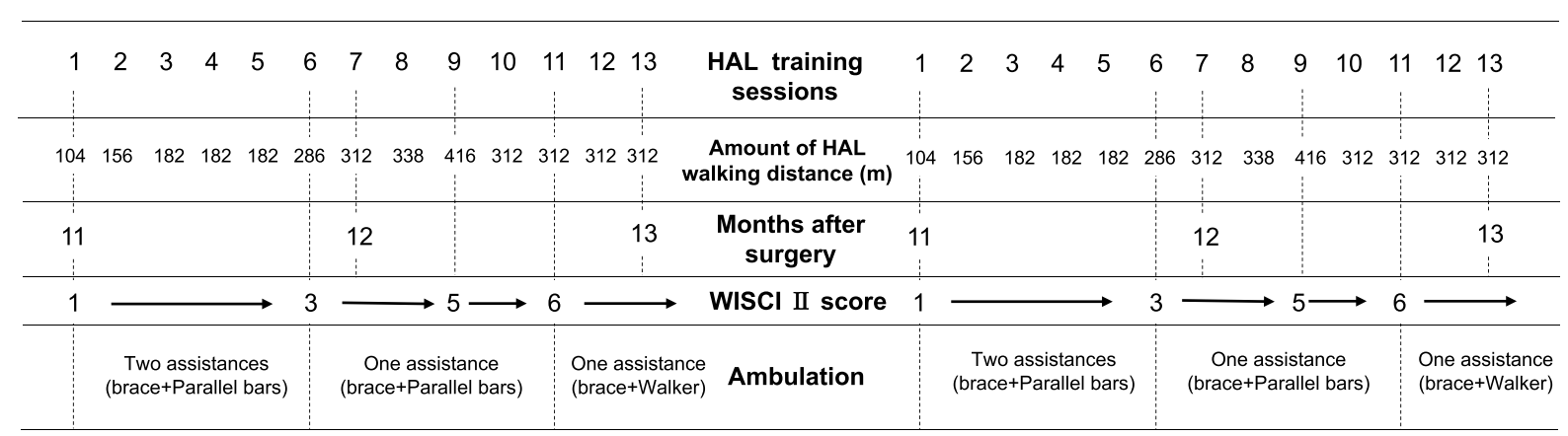

Figure 2 Entire process of the patient's hybrid assistive limb (HAL) gait training. The graph shows the chronological improvement in walking speed, step length, and cadence in the 10-meter walking test without the HAL during HAL gait sessions. Gait speed (A) as well as step length and cadence (B) improved in each HAL session. In the first nine sessions, the patient's walking ability (WISCl-II and ambulation) were gradually improved. After nine sessions, the patient could walk with one assistance and use brace and bilateral crutches. The walking training distance with the HAL also gradually increased. 
Table I Detailed Results of the ISNCSCI Motor Score (Upper and Lower Extremity) at Baseline (Pre-HAL) and After Training (Post-HAL)

\begin{tabular}{|c|c|c|c|c|c|}
\hline & \multicolumn{2}{|c|}{$\begin{array}{c}\text { Baseline } \\
\text { (Pre HAL) }\end{array}$} & \multicolumn{2}{|c|}{$\begin{array}{c}\text { After Training } \\
\text { (Post HAL) }\end{array}$} \\
\hline & & $\mathbf{R t}$ & Lt & $\mathbf{R t}$ & Lt \\
\hline C5 & Elbow Flexor & 5 & 5 & 5 & 4 \\
\hline C6 & Wrist Extensor & 5 & 4 & 5 & 4 \\
\hline C7 & Elbow Extensor & 4 & 2 & 5 & 2 \\
\hline C8 & Finger Flexors & I & I & 4 & 4 \\
\hline $\mathrm{TI}$ & Finger Abductor & 3 & 0 & 3 & 2 \\
\hline \multicolumn{2}{|c|}{ Total upper extremities } & \multicolumn{2}{|c|}{30} & \multicolumn{2}{|c|}{38} \\
\hline L2 & Hip Flexor & 3 & 3 & 4 & 4 \\
\hline L3 & Knee Extensor & 4 & 4 & 4 & 4 \\
\hline L4 & Ankle Dorsiflexor & 2 & 2 & 4 & 4 \\
\hline L5 & Long Toe Extensor & 4 & 2 & 4 & 4 \\
\hline SI & Ankle Plantar Flexor & 3 & 2 & 3 & 3 \\
\hline \multicolumn{2}{|c|}{ Total lower extremities } & \multicolumn{2}{|c|}{29} & \multicolumn{2}{|c|}{38} \\
\hline \multirow{2}{*}{\multicolumn{2}{|c|}{ Total }} & 34 & 25 & 41 & 35 \\
\hline & & \multicolumn{2}{|c|}{59} & \multicolumn{2}{|c|}{76} \\
\hline
\end{tabular}

which, the patient had never observed swelling and pain. We could not confirm whether the knee pain was attributed to the HAL training; however, none of the sessions needed to be postponed or cancelled due to knee pain.

\section{Discussion}

Our patient had shown significant improvement in functional walking ability, although it reached a plateau during the first 6 months after SCI. Furthermore, even though this patient was treated using conventional rehabilitation in another hospital, improvement in functional walking ability and other physical functions had not been observed. Surprisingly, we observed improvement in the patient's functions, especially walking ability, immediately after starting the HAL training. Other studies ${ }^{7}$ reported effects of HAL training in various chronic SCI cases classified by AIS; however, all the patients involved were able to walk and perform high-performance tests such as timed-up and go test, and 6-minute walk test, as opposed to our patient's walking ability. Moreover, our patient took a shorter-term HAL training compared to those of the previous report (our study: two or three times per week for 2 months, previous study: five times per week for 3 months).

There are several possible mechanisms underlying the significant HAL-induced neurological recovery in the present case of a patient with chronic SCI, in which recovery
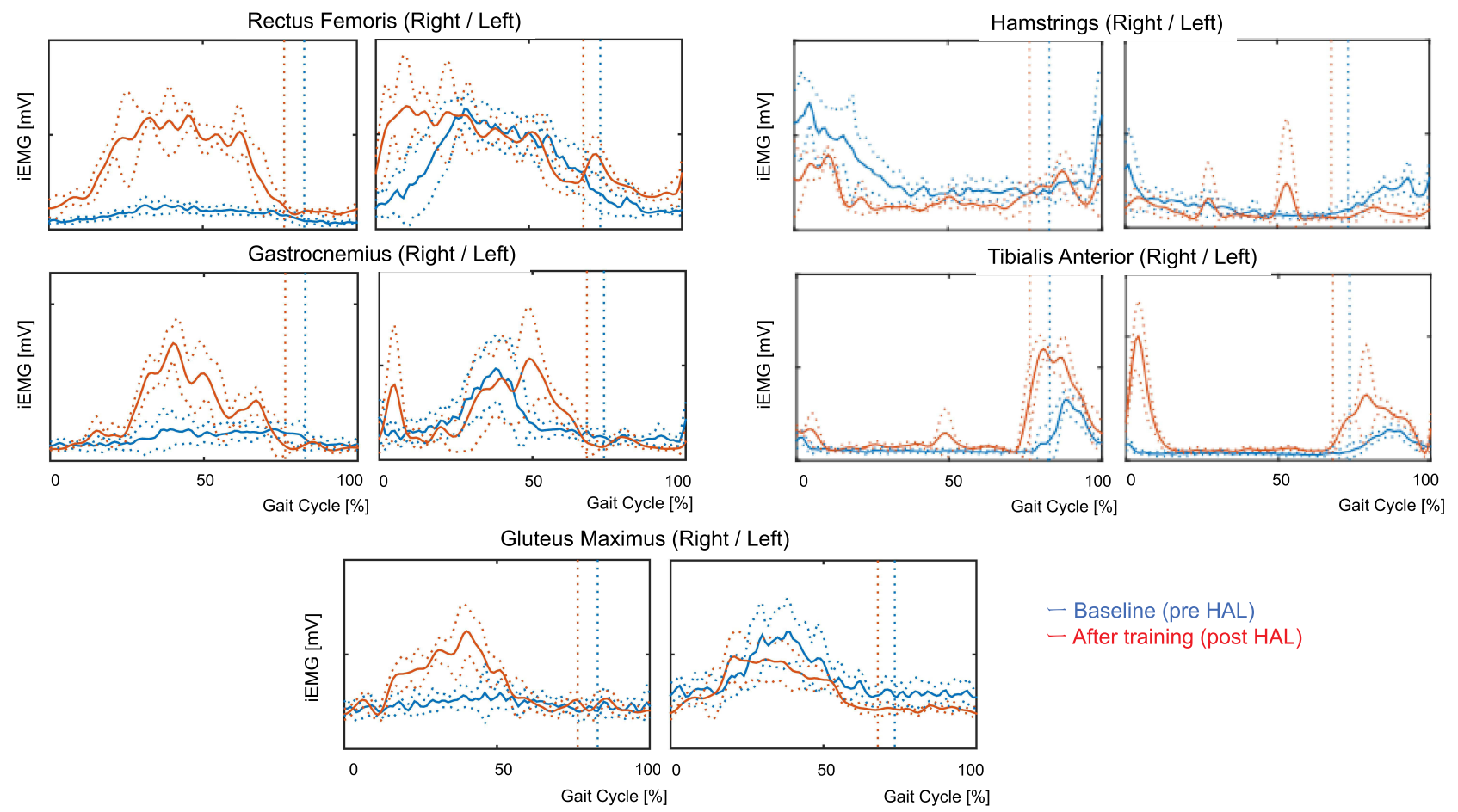

- Baseline (pre HAL)

- After training (post HAL)

Figure 3 Muscle activities of the rectus femoris, hamstrings, gastrocnemius, tibialis anterior and gluteus maximus without the hybrid assistive limb (HAL) at baseline (pre$\mathrm{HAL}$ ) and after training (post-HAL). Each muscle on the right side was activated in the stance phase after the HAL training. Vertical lines indicate the moment of toe lift. 

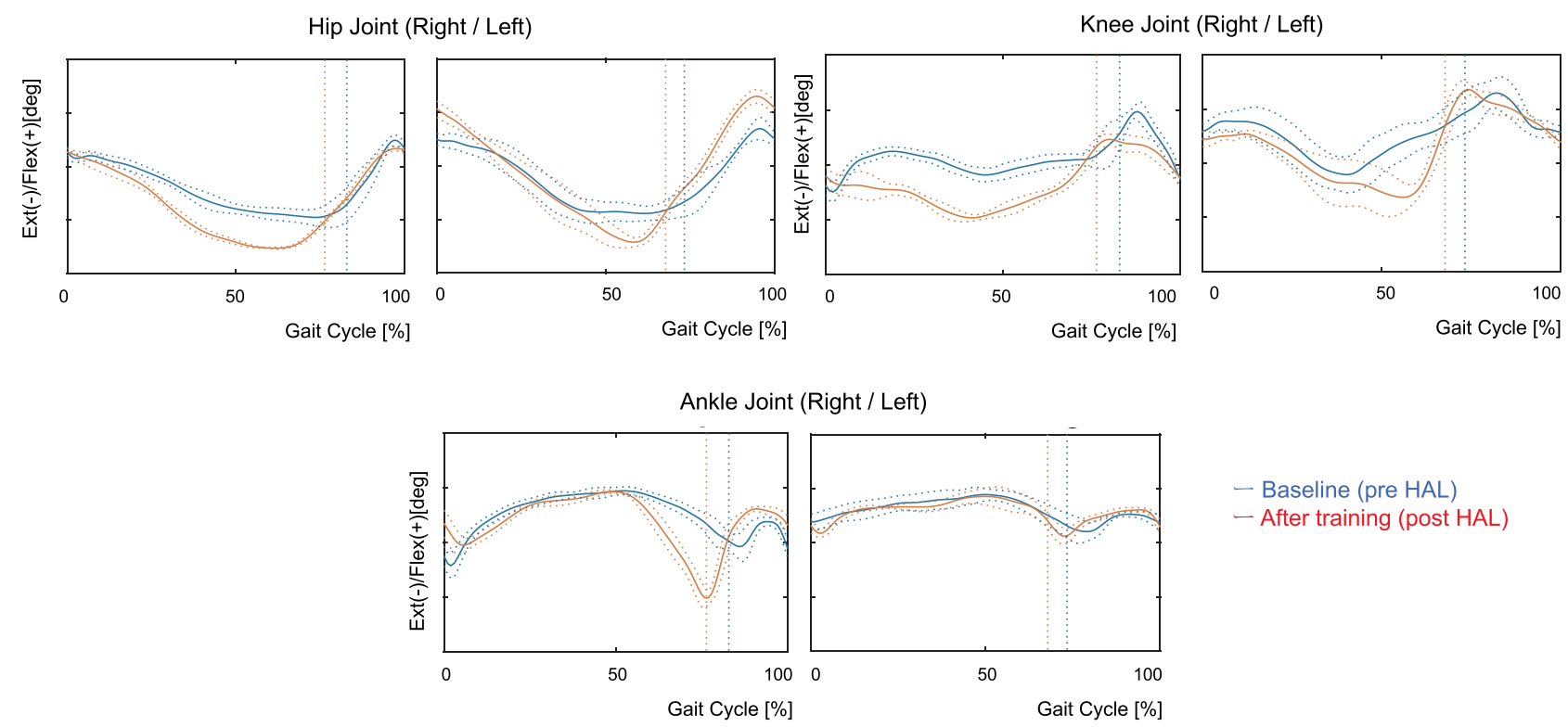

- Baseline (pre HAL)

- After training (post HAL)

Figure 4 Temporal profile of the angular position of the hip, knee, and ankle joint over the gait cycle, measured without the $\mathrm{HAL}$ at baseline (pre-HAL) and after training (post-HAL). After HAL training sessions, the maximum hip and knee extension angle on both sides during the stance phases increased. Moreover, the maximum right ankle plantar flexion angle during the stance phase increased post HAL. Vertical lines indicate the moment of toe lift.

Abbreviations: RF, rectus femoris; Gmax, gluteus maximus; GAS, gastrocnemius; HAL, hybrid assistive limb.

had reached a plateau during conventional rehabilitation. First, active subject participation in robot-driven gait therapy using HAL could lead to possible gains in motor learning. Second, regarding cortical reorganization, a previous study reported that the effect of active movement is better than that of passive movement. ${ }^{13}$ We consider that HAL training is more effective for its use of innate neuromuscular control, compared with other rehabilitation robots, such as the Lokomat and Rewalk, which do not include sensors to detect the neuromuscular activation of wearers. Therefore, HAL-assisted active motor training is more effective than passive motor training in eliciting performance improvements and cortical reorganization. HAL can assist the patient's lower limb motion only when the patient's muscles are active, and this assures the consistent signaling of sensorimotor interactions in the patient's central nervous system. Therefore, appropriate sensory inputs are essential to achieve maximum functional recovery in SCIs. Regarding the upper-extremity movement, improvement of ISNCSCI motor score was observed not only in lower-extremity score but also in upper-extremity score, even though the training using HAL was only gait training. Recent research has indicated that voluntary upper limb alternate movement generates locomotor-like movements ${ }^{14}$ and enhances muscle activation in the lower limbs. ${ }^{15}$ Therefore, we consider that gait training by HAL might attribute to the improvement of upper-extremity motor function through the enhancement of neural repair and plasticity due to the interaction between upper and lower limbs.

This study used surface electromyography in synchronization with a motion capture system before and after HAL training. Muscle activity of the right limb muscles was more activated after HAL training than before HAL training. The mean hip extension angle, knee extension angle, and ankle plantar flexion angle during the stance phases in the motion capture system were also increased after HAL training. Based on the results of EMG and Vicon, we conjectured that the HAL training that contributed to the improvement in the patient's walking abilities would allow close to normal walking due to the changed walking strategy and enhanced neural recovery of the lower muscles. Some previous studies have reported that HAL generates joint torques by amplifying the sensed muscle activity. ${ }^{16,17}$ The patient's ability to bear weight on his feet improved. We consider that HAL training with a sufficient amount of gait training might be used as a treatment for disuse syndrome in the patient. There might have been disuse of walking muscles because the present patient's symptoms were too severe to perform walking exercise in the other hospital. Because of the above reasons, we believe that HAL motion assistive technologies could contribute to improvement in patient walking ability by 
facilitating proper joint motion and loading and unloading muscle movement.

\section{Conclusion}

The gait training using HAL for chronic severe incomplete tetraplegic SCI has the potential to improve patients' ability to walk. Further exploration is needed to prove the beneficial effects of HAL gait training.

\section{Acknowledgments}

We thank Mayuko Sakamaki and Yumiko Ito, at the Center for Innovative Medicine and Engineering (CIME), University of Tsukuba Hospital, for their excellent technical assistance.

\section{Funding}

This work was supported by the Industrial Disease Clinical Research Grants of the Ministry of Health, Labour and Welfare Grant Number 14060101-01. This work was also was supported by the Grants-in-Aid for Encouragement of Scientists, Grant Number 20H01124.

\section{Disclosure}

Yoshiyuki Sankai reports he is an inventor of the world's first wearable cyborg HAL, a stockholder and the CEO of university venture company CYBERDYNE, the manufacturer of the wearable cyborg HAL. His conflicts of interest are strictly managed by the board of directors of CYBERDYNE, and by the University of Tsukuba according to the national university rules. Patents of HAL belong to University of Tsukuba, and CYBERDYNE pays a patent royalty to the University of Tsukuba according to national university rules. CYBERDYNE was not involved in the study design, in the collection, analysis, or interpretation of the data, in the writing of the report, in the financial/non-financial support, or in the decision to submit the paper for publication. The authors report no other conflicts of interest in this work.

\section{References}

1. Wyndaele M, Wyndaele JJ. Incidence, prevalence and epidemiology of spinal cord injury: what learns a worldwide literature survey? Spinal Cord. 2006;44:523-529. doi:10.1038/sj.sc.3101893

2. Fawcette JW, Curt A, Steeves JD, et al. Guidelines for the conduct of clinical trials for spinal cord injury as developed by the ICCP panel: spontaneous recovery after spinal cord injury and statistical power needed for therapeutic clinical trials. Spinal Cord. 2007;45(3):190205. doi:10.1038/sj.sc.3102007
3. Jm P, Jenkins NR. Late neurological changes following traumatic spinal cord injury. J Neurosurg. 1988;69:399-402. doi:10.3171/ jns.1988.69.3.0399

4. Esquenazi A, Talaty M. Robotics for lower limb rehabilitation. Phys Med Rehabil Clin N Am. 2019;30:385-397. doi:10.1016/j. pmr.2018.12.012

5. Kawamoto H, Sankai Y. Power assist method based on phase sequence and muscle force condition for HAL. Adv Robot. 2005;19 (7):717-734. doi:10.1163/1568553054455103

6. Wall A, Borg J, Palmcrantz S. Clinical application of the hybrid assistive limb (HAL) for gait training-a systematic review. Front Syst Neurosci. 2015;9:48. doi:10.3389/fnsys.2015.00048

7. Aach M, Cruciger O, Sczesny-Kaiser M, et al. Voluntary driven exoskeleton as a new tool for rehabilitation in chronic spinal cord injury: a pilot study. Spine J. 2014;14(12):2847-2853. doi:10.1016/j. spinee.2014.03.042

8. Jansen O, Grasmuecke D, Meindl RC, et al. Hybrid assistive limb exoskeleton HAL in the rehabilitation of chronic spinal cord injury: proof of concept; the results in 21 patients. World Neurosurg. 2018;110:e73-e78. doi:10.1016/j.wneu.2017.10.080

9. Kirshblum SC, Burns SP, Biering-Sørensen F, et al. International standards for neurological classification of spinal cord injury (revised 2011). J Spinal Cord Med. 2011;34:535-546. doi:10.1179/ 204577211X13207446293695

10. Kirshblum SC, Biering-Sørensen F, Betz R, et al. International standards for neurological classification of spinal cord injury: cases with classification chal- lenges. J Spinal Cord Med. 2014;37(2):120-127. doi:10.1179/2045772314Y.0000000196

11. Ditunno JF Jr, Ditunno PL, Scivoletto G, et al. The walking index for spinal cord injury (WISCI/WISCI II): nature, metric properties, use and misuse. Spinal Cord. 2013;51:346-355. doi:10.1038/ sc. 2013.9

12. Scivoletto G, Tamburella F, Laurenza L, Torre M, Molinari M, Ditunno JF. Walking index for spinal cord injury version II in acute spinal cord injury: reliability and reproducibility. Spinal Cord. 2014;52:65-69. doi:10.1038/sc.2013.127

13. Lotze M, Braun C, Birbaumer N, Anders S, Cohen LG. Motor learning elicited by voluntary drive. Brain. 2003;126:866-872. doi:10.1093/brain/awg079

14. Sylos-Labini F, Ivanenko YP, Maclellan MJ, et al. Locomotor-like leg movements evoked by rhythmic arm movements in humans. PLoS One. 2014;9:e90775. doi:10.1371/journal.pone.0090775

15. de Kam D, Rijken H, Manintveld T, et al. Arm movements can increase leg muscle activity during submaximal recumbent stepping in neurologically intact individuals. $J$ Appl Physiol. 2013;115 (1):34-42. doi:10.1152/japplphysiol.00510.2012

16. Kadone H, Kubota $\mathrm{S}$, Abe $\mathrm{T}$, et al. Muscular activity modulation during post-operative walking with hybrid assistive limb (HAL) in a patient with thoracic myelopathy due to ossification of posterior longitudinal ligament: a case report. Front Neurol. 2020;11:102. doi:10.3389/fneur.2020.00102

17. Kubota S, Abe T, Kadone H, et al. Hybrid assistive limb (HAL) treatment for patients with severe thoracic myelopathy due to ossification of the posterior longitudinal ligament (OPLL) in the postoperative acute/subacute phase: a clinical trial. J Spinal Cord Med. 2019;42:517-525. doi:10.1080/10790268.2018.1525975 


\section{Publish your work in this journal}

The International Medical Case Reports Journal is an international, peer-reviewed open-access journal publishing original case reports from all medical specialties. Previously unpublished medical posters are also accepted relating to any area of clinical or preclinical science. Submissions should not normally exceed 2,000 words or 4

published pages including figures, diagrams and references. The manuscript management system is completely online and includes a very quick and fair peer-review system, which is all easy to use. Visit http://www.dovepress.com/testimonials.php to read real quotes from published authors. 